\title{
Conditions for the Existence of Global Solutions to Doubly Nonlinear Advection-diffusion Equations
}

\author{
J. Q. CHAGAS ${ }^{1 *}$, P. L. GUIDOLIN ${ }^{2}$ and P. R. ZINGANO ${ }^{3}$
}

Received on December 10, 2018 / Accepted on September 27, 2019

\begin{abstract}
In this work, we consider a initial-value problem for an doubly nonlinear advection-diffusion equation, and we present a critical value of $\kappa$ up to wich the initial-value problem has global solution independent of the initial data $u_{0}$, and from which global solutions may still exists, but from initial data $u_{0}$ satisfying certain conditions. For this, we suppose that the function $\boldsymbol{f}(x, t, u)$ in the advection term, writted in the divergent form, satisfies certain conditions about your variation in $\mathbb{R}^{n}$, and we also use the decrease of the norm $L^{1}\left(\mathbb{R}^{n}\right)$ and an control for the norm $L^{\infty}\left(\mathbb{R}^{n}\right)$ of solution $u(\cdot, t)$.
\end{abstract}

Keywords: doubly nonlinear parabolic equation, global solutions, conditions for global solutions.

\section{INTRODUCTION}

We will considerer the regularized problem

$$
\left\{\begin{array}{l}
u_{t}+\operatorname{div}(\boldsymbol{f}(x, t, u))=\operatorname{div}\left(|u|^{\alpha}|\nabla u|^{\beta} \nabla u\right)+\eta \Delta u, \quad x \in \mathbb{R}^{n}, t>0, \\
u(\cdot, 0)=u_{0} \in L^{p_{0}}\left(\mathbb{R}^{n}\right) \cap L^{\infty}\left(\mathbb{R}^{n}\right)
\end{array}\right.
$$

where $\eta>0$ is fixed and $1 \leq p_{0}<\infty$ is given; $\alpha$ and $\beta$ are constants, with $\alpha, \beta \geq 0$ and $\alpha+\beta>0$; and the function $\boldsymbol{f}(x, t, u)$ satisfies

$$
|\boldsymbol{f}(x, t, u)| \leq B(T)|u|^{\kappa+1}
$$

for $\kappa \geq 0$ and for all $x \in \mathbb{R}^{n}, t \geq 0, u \in \mathbb{R}$, where $B(T)<\infty$ denotes the variation of $f(x, t, u)$ in $\mathbb{R}^{n}$, and controls the magnitude of its derivatives.

*Corresponding author: J.Q. Chagas - E-mail: jocemarchagas@ gmail.com

${ }^{1}$ Departamento de Matemática e Estatística, UEPG, Av. General Carlos Cavalcanti, 4748, 84030-900, Ponta Grossa, PR, Brasil. E-mail: jocemarchagas@uepg.br https://orcid.org/0000-0001-6828-3340

2 Departamento de Matemática Pura e Aplicada, UFRGS, Av. Bento Gonçalves, 9500, 91509-900, Porto Alegre, RS, Brasil. E-mail: patiguidolin@gmail.com https://orcid.org/0000-0002-1609-6073

3 Departamento de Matemática Pura e Aplicada, UFRGS, Av. Bento Gonçalves, 9500, 91509-900, Porto Alegre, RS, Brasil. E-mail: paulo.zingano@ufrgs.br https://orcid.org/0000-0002-5074-9146 
As solution to the regularized problem (1.1) in a determined interval $[0, T]$, for $0<T<T_{*} \leq$ $\infty$, we consider a function $u(\cdot, t) \in L^{\infty}\left([0, T], L^{\infty}\left(\mathbb{R}^{n}\right)\right)$, smooth, which solves the equation in (1.1) in the classic sense for $0 \leq t \leq T<T_{*}$, and satisfies the initial condition in the sense of $L^{p_{0}}\left(\mathbb{R}^{n}\right)$ when $t \rightarrow 0$. The $\left[0, T_{*}\right)$ interval is known as the maximal interval for the solution, and the existence of such $T_{*}$ is guaranteed by general theory of Parabolic Equations (see, e.g., [7] or [8]), so that the local existence of solutions for the problem (1.1) is guaranteed.

If we consider the additional stability condition

$$
\sum_{i=1}^{n} u \frac{\partial f}{\partial x_{i}}(x, t, u) \geq 0
$$

then we can obtain for the solutions of (1.1) the following fundamental supnorm estimate:

$$
\|u(\cdot, t)\|_{L^{\infty}\left(\mathbb{R}^{n}\right)} \leq K(n, p)\left\|u_{0}\right\|_{L^{p}\left(\mathbb{R}^{n}\right)}^{\rho} t^{-\sigma}, \quad \forall t>0,
$$

where the values of $\rho$ and $\sigma$ are $\rho=\frac{p(\beta+2)}{n(\alpha+\beta)+p(\beta+2)}$ and $\sigma=\frac{n}{n(\alpha+\beta)+p(\beta+2)}$. Thereby, it is easy to see that the solution has global existence, and more: the solution goes to the zero when $t \rightarrow \infty$ (see [2]).

However, in the general case, the existence of global solutions is not easy to obtain. The central question is that in the search for conditions that guarantee the global existence of solutions, it is fundamental that we control the high norms of the solutions, especially the supnorm (see, e.g., [7]). In general situations, the task of controlling $\|u(\cdot, t)\|_{L^{\infty}\left(\mathbb{R}^{n}\right)}$ can become very difficult. To illustrate this question, intuitively, let us consider positive solutions of the following equation, simpler than that of the problem (1.1): $u_{t}+\left(b(x) u^{2}\right)_{x}=\left(u^{\alpha}\left|u_{x}\right|^{\beta} u_{x}\right)_{x}, x \in \mathbb{R}, t>0$, that we can rewrite as $u_{t}+2 b(x) u u_{x}=\left(u^{\alpha}\left|u_{x}\right|^{\beta} u_{x}\right)_{x}-u^{2} \frac{d}{d x} b(x)$. In this equation, the dissipative term tends to make the magnitude of the solution decrease, but the term $-u^{2} \frac{d}{d x} b(x)$, in regions where $\frac{d}{d x} b(x)$ is negative, tends to make the magnitude of the solution increase. The result of this competition isn't easy to predict.

In [3] we considered more general conditions for the function $\boldsymbol{f}(x, t, u)$ and obtain for solutions of (1.1), for all $t>0$, the following supnorm estimate:

$$
\|u(\cdot, t)\|_{L^{\infty}\left(\mathbb{R}^{n}\right)} \leq K(n, p, \alpha, \beta, \kappa) \cdot \max \left\{\left\|u\left(\cdot, t_{0}\right)\right\|_{L^{\infty}\left(\mathbb{R}^{n}\right)} ;\left(\mathbb{B}\left(t_{0} ; t\right)\right)^{\delta_{1}}\left(\mathbb{U}_{p}\left(t_{0} ; t\right)\right)^{\delta_{2}}\right\}
$$

with $\delta_{1}=\frac{n}{n(\alpha+\beta-\kappa)+p(\beta+1)}$ e $\delta_{2}=\frac{p(\beta+1)}{n(\alpha+\beta-\kappa)+p(\beta+1)}$, where $\mathbb{B}\left(t_{0} ; t\right):=\sup _{t_{0} \leq \tau \leq t}(B(\tau))$, for $0 \leq t_{0} \leq$ $t<T_{*}$, and $\mathbb{U}_{p}\left(t_{0} ; t\right):=\sup _{t_{0} \leq \tau \leq t}\left(\|u(\cdot, \tau)\|_{L^{p}\left(\mathbb{R}^{n}\right)}\right)$, for $1 \leq p_{0} \leq p \leq \infty$. This controls the supnorm, but it is does not guarantee the existence of global solutions to (1.1), for $1 \leq p_{0} \leq p \leq \infty$. In this work, we will consider $p_{0}=1$ and we will present conditions over $\kappa$ with which the existence of global solution is guaranteed.

The equation in (1.1) generalizes several important equations, including the Porous Media Equation $(\beta=0)$ and the $p$-Laplacian Equation ( $\alpha=0$ and $\beta=p-2)$; and is considered, for example, 
in [4] and in [9]. The basic ideas used in our procedure can be seen in [5], where they are apllied to a somewhat simpler equation and where the additional condition (1.3) is satisfied; and the ideas presented in this paper can be seen in more detail in [1].

\section{HYPOTHESES AND PRELIMINARY RESULTS}

In this Section we rank the preliminary results to be used in the proof of the major result of this paper: the Theorem 2.3, presented in Section 3.

We begin presenting some reasonable hypothesis about the function $\boldsymbol{f}(x, t, u)$ and about the solution $u(\cdot, t)$ of (1.1), for $t \in[0, T]$ and $T<T_{*}$, that we use to obtain our results.

Consider $\boldsymbol{f}(x, t, u)=\boldsymbol{b}(x, t, u) u$, where $\boldsymbol{b}(x, t, u)$ is a smooth function satisfying $|\boldsymbol{b}(x, t, u)| \leq$ $B(t)|u|^{\kappa}, \forall x \in \mathbb{R}^{n}, \forall t \geq 0, \forall u \in \mathbb{R}$, such that $f(x, t, u)$ satisfies (1.2). $B \in C^{0}([0, \infty))$ denotes the variation in $\boldsymbol{b}(x, t, u)$ in $\mathbb{R}^{n}$ : for each $1 \leq i \leq n$ we become $B_{i}(t)$ by

$$
B_{i}(t):=\frac{1}{2}\left\{\sup _{x \in \mathbb{R}^{n}} b_{i}(x, t, u(x, t))-\inf _{x \in \mathbb{R}^{n}} b_{i}(x, t, u(x, t))\right\}, \forall 0 \leq t<T_{*},
$$

and therefore $B(t):=\left|\left(B_{1}(t), \cdots, B_{n}(t)\right)\right|_{2}$ for each $0 \leq t<T_{*}$. Besides, we require that $\boldsymbol{b}(x, t, u)$ satisfies $\boldsymbol{b}, \boldsymbol{b}_{x_{1}}, \cdots, \boldsymbol{b}_{x_{n}}, \boldsymbol{b}_{u} \in C^{0}\left(\mathbb{R}^{n} \times[0, \infty) \times \mathbb{R}\right)$.

As the solution $u(\cdot, t)$ to (1.1) is limited for each $0 \leq t \leq T<T_{*}$, we consider the following limitation estimate: $\|u(\cdot, t)\|_{L^{\infty}\left(\mathbb{R}^{n}\right)} \leq M(T), \forall 0 \leq t \leq T$. We also assume the convergence of the solution to the given initial value $u_{0}$ as $t \rightarrow 0$, is in the sense of $L^{p_{0}}\left(\mathbb{R}^{n}\right)$. In addition, we suppose that $\nabla u \in L^{\infty}\left(\mathbb{R}^{n} \times\left[t_{0}, T\right]\right)$ for each $0<t_{0}<T$ and, therefore, for each $t_{0} \leq t \leq T$ we have an estimate in the form $|\nabla u(x, t)| \leq C\left(t_{0}\right)$, for all $0<t_{0} \leq T$.

The first result in this section is specific for $p_{0}=1$ in (1.1): it is verified that, for $[0, T]$ in the maximal interval of existence, the solutions $u(\cdot, t)$ have decreasing $L^{1}\left(\mathbb{R}^{n}\right)$, more specifically:

Theorem 2.1. Let $u(\cdot, t) \in L^{\infty}\left([0, T], L^{\infty}\left(\mathbb{R}^{n}\right)\right)$ be a solution for (1.1) with $p_{0}=1$, for $0<T<$ $T_{*} \leq \infty$. Then,

$$
\|u(\cdot, t)\|_{L^{1}\left(\mathbb{R}^{n}\right)} \leq\left\|u\left(\cdot, t_{0}\right)\right\|_{L^{1}\left(\mathbb{R}^{n}\right)}, \quad \forall 0 \leq t_{0} \leq t \leq T .
$$

In particular, when $t_{0}=0$,

$$
\|u(\cdot, t)\|_{L^{1}\left(\mathbb{R}^{n}\right)} \leq\|u(\cdot, 0)\|_{L^{1}\left(\mathbb{R}^{n}\right)}, \quad \forall 0 \leq t \leq T .
$$

As well as the norm $L^{1}(\mathbb{R})$ decrease, also the properties of contraction in $L^{1}\left(\mathbb{R}^{n}\right)$, mass conservation and comparison principle are valid. The proofs of these properties, including Theorem 2.1, can be found in [1], but there's no novelty in these properties as they are already known and plenty of proofs can be found in the literature.

In [3] we present the estimate (1.4) for the norm of the sup for the solutions of (1.1), valid for $1 \leq p_{0} \leq p \leq \infty$, and the proof is outlined in the following. We begin with an important energy inequality: 
Theorem 2.2. Let $u(\cdot, t) \in L_{\text {loc }}^{\infty}\left(\left[0, T_{*}\right), L^{\infty}\left(\mathbb{R}^{n}\right)\right)$ solution of (1.1) for $0 \leq t<T_{*}$. Suppose $u \in$ $L^{p}\left(\mathbb{R}^{n}\right)$ for some $p \geq p_{0}$, then

$$
\begin{aligned}
\frac{d}{d t}\|u(\cdot, t)\|_{L^{q\left(\mathbb{R}^{n}\right)}}^{q} & +q(q-1) \int_{\mathbb{R}^{n}}|u(x, t)|^{q-2+\alpha}|\nabla u(x, t)|^{\beta+2} d x \leq \\
& \leq q(q-1) \int_{\mathbb{R}^{n}}|u(x, t)|^{q-2} \nabla u(x, t) \cdot \boldsymbol{f}(x, t, u) d x,
\end{aligned}
$$

for all $q$ satisfying $q \geq p \geq p_{0}$ and $q \geq 2$, and for all $t \in\left(0, T_{*}\right) \backslash E_{q}$, where $E_{q} \subset(0, \infty)$ is a null set.

Proof. See [3].

Then, for $p \geq p_{0}$, the hypothesis $p_{0} \leq p \leq q$ is changed to $\sigma p \leq q<\infty$, where $\sigma$ satisfies $\sigma \geq 1$ and $\sigma \geq 1+\frac{\gamma_{-}}{p}$, with $\gamma_{-}$denoting the negative part of $\gamma=\frac{\kappa(\beta+2)-(\alpha+\beta)}{(\beta+1)}$. p must satisfy, additionaly, the condition $p>\frac{n(\kappa-(\alpha+\beta))}{(\beta+1)}$. Together with the energy inequality (2.2), we use the interpolation inequality of Sobolev-Nirenberg-Gagliardo (SNG) type:

$$
\|w(\cdot, t)\|_{L^{r}\left(\mathbb{R}^{n}\right)} \leq C\|w(\cdot, t)\|_{L^{s}\left(\mathbb{R}^{n}\right)}^{1-\theta}\|\nabla w(\cdot, t)\|_{L^{\widetilde{p}}\left(\mathbb{R}^{n}\right)}^{\theta}, \quad \forall w \in C_{0}^{1}\left(\mathbb{R}^{n}\right),
$$

where $0 \leq \theta \leq 1$, and $r, s$ and $\widetilde{p}$ satisfies $0<s \leq r \leq \infty, 1 \leq \widetilde{p} \leq \infty$, and $\frac{1}{r}=\left(\frac{1}{\tilde{p}}-\frac{1}{n}\right) \theta+\frac{(1-\theta)}{s}$ (for more details about this inequality, see, for instance, [6]), to prove what we call Fundamental Lemma, a result relating the norms $L^{q}$ and $L^{q / \sigma}$ of the solutions $u(\cdot, t)$ :

Lemma 2.1. (Fundamental Lemma) Let $u(\cdot, t) \in L_{\text {loc }}^{\infty}\left(\left[0, T_{*}\right), L^{\infty}\left(\mathbb{R}^{n}\right)\right)$ solution of $(1.1)$ for $0 \leq$ $t<T_{*}$. If $q$ satisfies $q \geq 2$ and $\sigma p \leq q<\infty$, then, for each $0 \leq t_{0}<T_{*}$,

$$
\mathbb{U}_{q}\left(t_{0} ; t\right) \leq \max \left\{\left\|u\left(\cdot, t_{0}\right)\right\|_{L^{q}\left(\mathbb{R}^{n}\right)} ; K(q) \mathbb{B}\left(t_{0} ; t\right)^{\frac{n(\sigma-1)}{(\beta+1)(q-\sigma a)}} \mathbb{U}_{q / \sigma}\left(t_{0} ; t\right)^{\frac{(q-a)}{(q-\sigma a)}}\right\},
$$

where $a=\frac{n[\kappa-(\alpha+\beta)]}{(\beta+1)}$ and $K(q)=\left(\frac{(q+\alpha+\beta)}{(\beta+2)}\right)^{\frac{n(\sigma-1)}{q-\sigma a}}\left(C_{1}\right)^{\frac{(q+\gamma)}{(q+\alpha+\beta)} \frac{n(\sigma-1)}{(q-\sigma a)}}\left(C_{2}\right)^{\frac{(\beta+2)}{(q+\alpha+\beta)}}$.

Proof: See [3].

The Fundamental Lemma is used in an iterative process to obtain, after a few steps, the estimate (1.4) for the limitation of the norm of the sup of the solution $u(\cdot, t)$ of $(1.1)$, for $0<t<T_{*} \leq \infty$ :

Theorem 2.3. Let $u(\cdot, t) \in L_{\text {loc }}^{\infty}\left(\left[0, T_{*}\right), L^{\infty}\left(\mathbb{R}^{n}\right)\right)$ solution of $(1.1)$ for $0 \leq t<T_{*}$. Given $p \geq p_{0}$, for each $0 \leq t_{0}<t<T_{*}$,

$$
\|u(\cdot, t)\|_{L^{\infty}\left(\mathbb{R}^{n}\right)} \leq K(n, p, \alpha, \beta, \kappa) \max \left\{\left\|u\left(\cdot, t_{0}\right)\right\|_{L^{\infty}\left(\mathbb{R}^{n}\right)} ;\left(\mathbb{B}\left(t_{0} ; t\right)\right)^{\delta_{1}}\left(\mathbb{U}_{p}\left(t_{0} ; t\right)\right) \delta_{2}\right\}
$$

where $\delta_{1}=\frac{n}{n(\alpha+\beta-\kappa)+p(\beta+1)}$ and $\delta_{2}=\frac{p(\beta+1)}{n(\alpha+\beta-\kappa)+p(\beta+1)}$.

Proof. In the first step we use the iterative argument that allows to estimate the norms $L^{q}$ of the solution $u(\cdot, t)$ for large values of $q$, in the interval $\left(t_{0}, t\right)$, with $0 \leq t_{0} \leq t \leq T_{*}$, as a function of 
lower norms of $u$ : we show that, for $u(\cdot, t) \in L_{l o c}^{\infty}\left(\left[0, T_{*}\right), L^{\infty}\left(\mathbb{R}^{n}\right)\right)$ solution to (1.1), given $p \geq p_{0}$, for each $0 \leq t_{0}<t<T_{*}$,

$$
\mathbb{U}_{\sigma p}\left(t_{0} ; t\right) \leq \max \left\{\left\|u\left(\cdot, t_{0}\right)\right\|_{L^{\sigma p}\left(\mathbb{R}^{n}\right)} ; K(\sigma p) \widetilde{\mathbb{B}}\left(t_{0} ; t\right)^{\frac{1}{(\sigma p-\sigma a)}} \mathbb{U}_{p}\left(t_{0} ; t\right)^{\frac{(\sigma p-a)}{(\sigma p-\sigma a)}}\right\},
$$

where $\widetilde{\mathbb{B}}\left(t_{0} ; t\right):=\mathbb{B}\left(t_{0} ; t\right)^{\frac{n(\sigma-1)}{(\beta+1)}}$. Besides, for each $m \geq 2$,

$$
\begin{aligned}
& \mathbb{U}_{\sigma^{m} p}\left(t_{0} ; t\right) \leq \max \left\{\left\|u\left(\cdot, t_{0}\right)\right\|_{L^{\sigma^{m} p\left(\mathbb{R}^{n}\right)}} ;\right. \\
& \left(\prod_{i=l}^{m}\left(K\left(\sigma^{i} p\right)\right)^{\frac{\sigma^{m} p-a}{\sigma^{m} p-\sigma^{m-i} a}}\right)\left(\widetilde{\mathbb{B}}\left(t_{0} ; t\right)\right)^{\sum_{i=l}^{m} \frac{\left(\sigma^{m} p-a\right)}{\sigma^{m-i+1}\left(\sigma^{i} p-a\right)\left(\sigma^{i-1} p-a\right)}}\left\|u\left(\cdot, t_{0}\right)\right\|_{L^{\sigma^{l-1} p\left(\mathbb{R}^{n}\right)}}^{\frac{\sigma^{m} p-a}{\sigma^{m}-\sigma^{m-l+1}}} \\
& \left.\left(\prod_{i=1}^{m}\left(K\left(\sigma^{i} p\right)\right)^{\frac{\sigma^{m} p-a}{\sigma^{m} p-\sigma^{m-i} a}}\right)\left(\widetilde{\mathbb{B}}\left(t_{0} ; t\right)\right)^{\sum_{i=1}^{m} \frac{\left(\sigma^{m} p-a\right)}{\sigma^{m-i+1}\left(\sigma^{i} p-a\right)\left(\sigma^{i-1} p-a\right)}}\left(\mathbb{U}_{p}\left(t_{0} ; t\right)\right)^{\frac{\sigma^{m} p-a}{\sigma^{m} p-\sigma^{m} a}}\right\}
\end{aligned}
$$

with $2 \leq l \leq m$. The proof of (2.6) is done by induction, taking $q=\sigma p$ in (2.4); then taking $q=\sigma^{2} p$ in (2.4), and likewise thereafter. Through simple manipulations of exponents, we obtain that, for each $0 \leq t_{0}<t<T_{*}$

$$
\begin{gathered}
\mathbb{U}_{\sigma^{m} p}\left(t_{0} ; t\right) \leq \max \left\{\left\|u\left(\cdot, t_{0}\right)\right\|_{L^{\sigma^{m}} p\left(\mathbb{R}^{n}\right)}\right. \\
\left(\prod_{i=l}^{m}\left(K\left(\sigma^{i} p\right)\right)^{\frac{\left(p-\sigma^{-m} a\right)}{\left(p-\sigma^{-i} a\right)}}\right)\left(\mathbb{B}\left(t_{0} ; t\right)\right)^{\frac{n}{(\beta+1)} \frac{\left(\sigma^{-l+1}-\sigma^{-m}\right)}{\left(p-\sigma^{-l+1} a\right)}}\left\|u\left(\cdot, t_{0}\right)\right\|_{L^{\sigma^{l-1} p\left(\mathbb{R}^{n}\right)}}^{\frac{\left(p-\sigma^{-m} a\right)}{(p-1 a)}} \\
\left.\left(\prod_{i=1}^{m}\left(K\left(\sigma^{i} p\right)\right)^{\frac{\left(p-\sigma^{-m} a\right)}{\left(p-\sigma^{-i} a\right)}}\right)\left(\mathbb{B}\left(t_{0} ; t\right)\right)^{\frac{n}{(\beta+1)} \frac{\left(1-\sigma^{-m}\right)}{(p-a)}}\left(\mathbb{U}_{p}\left(t_{0} ; t\right)\right)^{\frac{\left(p-\sigma^{-m} p\right)}{(p-a)}}\right\}
\end{gathered}
$$

for $2 \leq l \leq m$. Besides, defining $C(l, m):=\prod_{i=l}^{m}\left(K\left(\sigma^{i} p\right)\right)^{\frac{\left(p-\sigma^{-m} a\right)}{\left(p-\sigma^{-i} a\right)}}$, we rewrite (2.7), for each $0 \leq t_{0}<t<T_{*}$, as

$$
\begin{aligned}
& \mathbb{U}_{\sigma^{m} p}\left(t_{0} ; t\right) \leq \max \left\{\left\|u\left(\cdot, t_{0}\right)\right\|_{L^{\sigma^{m} p\left(\mathbb{R}^{n}\right)}} ;\right. \\
& (C(l, m))\left(\mathbb{B}\left(t_{0} ; t\right)\right)^{\frac{n}{(\beta+1)}} \frac{\left(\sigma^{-l+1}-\sigma^{-m}\right)}{\left(p-\sigma^{-l+1} a\right)}\left\|u\left(\cdot, t_{0}\right)\right\|_{L^{\sigma^{-1} p\left(\mathbb{R}^{n}\right)}}^{\left.\frac{\left(p-\sigma^{-m} a\right)}{(p-l+1}\right)}, \quad \forall 2 \leq l \leq m ; \\
& \left.(C(1, m))\left(\mathbb{B}\left(t_{0} ; t\right)\right)^{\frac{n}{(\beta+1)}} \frac{\left(1-\sigma^{-m}\right)}{(p-a)}\left(\mathbb{U}_{p}\left(t_{0} ; t\right)\right)^{\frac{\left(p-\sigma^{-m} p\right)}{(p-a)}}\right\} .
\end{aligned}
$$

Next, using the inequality of norms interpolation $\|u\|_{L^{s}\left(\mathbb{R}^{n}\right)} \leq\|u\|_{L^{r}\left(\mathbb{R}^{n}\right)}^{(1-\theta)}\|u\|_{L^{\widetilde{p}}\left(\mathbb{R}^{n}\right)}^{\theta}$, where $r<s<\widetilde{p}$ and $0 \leq \theta \leq 1$ satisfy $\frac{1}{s}=\frac{(1-\theta)}{r}+\frac{\theta}{\widetilde{p}}$, we obtain a simpler estimate for $\mathbb{U}_{\sigma^{m} p}\left(t_{0} ; t\right)$ by estimating the intermediate terms of (2.8) appropriately and putting toghether with the first and last terms: we prove that, given $p \geq p_{0}$, for each $0 \leq t_{0}<t<T_{*}$,

$$
\mathbb{U}_{\sigma^{m} p}\left(t_{0} ; t\right) \leq \widetilde{K}(m) \max \left\{\left\|u\left(\cdot, t_{0}\right)\right\|_{L^{\sigma^{m} p\left(\mathbb{R}^{n}\right)}} ;\left(\mathbb{B}\left(t_{0} ; t\right)\right)^{\frac{n}{(\beta+1)}} \frac{\left(1-\sigma^{-m}\right)}{(p-a)}\left(\mathbb{U}_{p}\left(t_{0} ; t\right)\right)^{\frac{\left(p-\sigma^{-m} p\right)}{(p-a)}}\right\}
$$

where $\widetilde{K}(m ; n, p, \alpha, \beta, \kappa)=\max \left\{1 ; \max _{1 \leq l \leq m} C(l, m)\right\}$. 
Simply by letting $m \rightarrow \infty$ in (2.9), we obtain the following estimate for the limitation of the norm of the sup of the solution $u(\cdot, t)$ for (1.1): given $p \geq p_{0}$, for each $0 \leq t_{0}<t<T_{*}$,

$$
\begin{aligned}
& \mathbb{U}_{\infty}\left(t_{0} ; t\right) \leq \widetilde{K}(n, p, \alpha, \beta, \kappa) \max \left\{\left\|u\left(\cdot, t_{0}\right)\right\|_{L^{\infty}\left(\mathbb{R}^{n}\right)} ;\right. \\
&\left.\left(\mathbb{B}\left(t_{0} ; t\right)\right)^{\frac{n}{n(\alpha+\beta-\kappa)+p(\beta+1)}}\left(\mathbb{U}_{p}\left(t_{0} ; t\right)\right)^{\frac{p(\beta+1)}{n(\alpha+\beta-\kappa)+p(\beta+1)}}\right\},
\end{aligned}
$$

Finaly, to obtain (2.5), we only recall the definition of $\mathbb{U}_{p}\left(t_{0} ; t\right)$, with $p=\infty$, and define $\delta_{1}$ and $\delta_{2}$, respectively, as:

$$
\delta_{1}=\frac{n}{n(\alpha+\beta-\kappa)+p(\beta+1)} ; \quad \delta_{2}=\frac{p(\beta+1)}{n(\alpha+\beta-\kappa)+p(\beta+1)} .
$$

\section{MAIN RESULT}

In this section we apply the results listed in Section 2 to obtain conditions for the global existence (i.e., conditions that guarantee that $T_{*}=\infty$ ) of solutions $u(\cdot, t)$ of $(1.1)$, with $p_{0}=1$, i.e.,

$$
\left\{\begin{array}{l}
u_{t}+\operatorname{div}(\boldsymbol{f}(x, t, u))=\operatorname{div}\left(|u|^{\alpha}|\nabla u|^{\beta} \nabla u\right)+\eta \Delta u, \quad x \in \mathbb{R}^{n}, t>0 \\
u(\cdot, 0)=u_{0} \in L^{1}\left(\mathbb{R}^{n}\right) \cap L^{\infty}\left(\mathbb{R}^{n}\right)
\end{array}\right.
$$

The first step is to obtain an energy inequality in an adequate form for the application.

Lemma 3.2. Let $u(\cdot, t) \in L_{\text {loc }}^{\infty}\left(\left[0, T_{*}\right), L^{\infty}\left(\mathbb{R}^{n}\right)\right)$ be a solution to (3.1) for $0 \leq t<T_{*}$. Suppose $u \in L^{p}\left(\mathbb{R}^{n}\right)$ for some $p \geq 1$,

$$
\begin{aligned}
& \frac{d}{d t}\|u(\cdot, t)\|_{L^{q}\left(\mathbb{R}^{n}\right)}^{q}+q(q-1) \int_{\mathbb{R}^{n}}|u(x, t)|^{q-2+\alpha}|\nabla u(x, t)|^{\beta+2} d x \leq \\
& \leq q(q-1) B(t) \frac{(\beta+2)}{(q+\alpha+\beta)} C^{(\beta+1) \frac{(q+\gamma)}{(q+\alpha+\beta)}}\|u(\cdot, t)\|_{L^{q / \sigma}\left(\mathbb{R}^{n}\right)} \frac{\left.1 \frac{1}{n} \frac{[(q+\kappa)(\beta+1)+(n-1)(\alpha+\beta-\kappa)]}{n}\right) q(\sigma-\sigma(\alpha+\beta)]}{\left[\left(\beta-1+\frac{(\beta+2)}{n}\right.\right.} . \\
& \cdot\left(\left(\frac{(q+\alpha+\beta)}{(\beta+2)}\right)^{(\beta+2)} \int_{\mathbb{R}^{n}}|u(x, t)|^{q-2+\alpha}|\nabla u(x, t)|^{\beta+2} d x\right) \sqrt[\left[\left(\sigma-1+\frac{1}{n}\right) q+\sigma \kappa\right]]{\left[\left(\sigma-1+\frac{(\beta+2)}{n}\right) q+\sigma(\alpha+\beta)\right]}
\end{aligned}
$$

for all $q$ satisfying $q>\sigma p$ and $q \geq 2$, and for all $t \in\left(0, T_{*}\right) \backslash E_{q}$, where $E_{q} \subset(0, \infty)$ is a null set.

Proof. We start with the energy inequality presented in (2.2). By taking an upper limit to the right-hand side and using Hölder, we obtain

$$
\begin{aligned}
& \frac{d}{d t}\|u(\cdot, t)\|_{L^{q\left(\mathbb{R}^{n}\right)}}^{q}+q(q-1) \int_{\mathbb{R}^{n}}|u(x, t)|^{q-2+\alpha}|\nabla u(x, t)|^{\beta+2} d x \leq \\
& \leq q(q-1) B(t)\left(\int_{\mathbb{R}^{n}}|u(x, t)|^{q-2+\alpha}|\nabla u(x, t)|^{\beta+2} d x\right)^{\frac{1}{\beta+2}}\left(\int_{\mathbb{R}^{n}}|u(x, t)|^{q+\gamma} d x\right)^{\frac{\beta+1}{\beta+2}},
\end{aligned}
$$


where $\gamma=\frac{\kappa(\beta+2)-(\alpha+\beta)}{(\beta+1)}$.

Defining $w(x, t) \in L^{1}\left(\mathbb{R}^{n}\right) \cap L^{\infty}\left(\mathbb{R}^{n}\right)$ by $w(x, t):=|u(x, t)|^{\lambda_{1}}$, for all $x \in \mathbb{R}^{n}, t>0$, where $\lambda_{1}=$ $\frac{q+\alpha+\beta}{(\beta+2)}$, we rewrite (3.3) as

$$
\begin{aligned}
& \frac{d}{d t}\|w(\cdot, t)\|_{L^{\lambda}\left(\mathbb{R}^{n}\right)}^{\lambda}+q(q-1)\left(\frac{(\beta+2)}{(q+\alpha+\beta)}\right)^{(\beta+2)}\|\nabla w(\cdot, t)\|_{L^{\beta+2}\left(\mathbb{R}^{n}\right)}^{(\beta+2)} \leq \\
& \leq q(q-1) B(t)\left(\frac{(\beta+2)}{(q+\alpha+\beta)}\right)\|\nabla w(\cdot, t)\|_{L^{\beta+2}\left(\mathbb{R}^{n}\right)}\|w(\cdot, t)\|_{L^{\tilde{\lambda}}\left(\mathbb{R}^{n}\right)}^{\tilde{\lambda} \frac{(\beta+2)}{(\beta+2)}}
\end{aligned}
$$

where $\lambda, \tilde{\lambda}$ e $\lambda_{0}$ are, respectivaly, $\lambda=\frac{q(\beta+2)}{(q+\alpha+\beta)} ; \tilde{\lambda}=\frac{(q+\gamma)(\beta+2)}{(q+\alpha+\beta)} ;$ and $\lambda_{0}=\frac{q(\beta+2)}{\sigma(q+\alpha+\beta)}$, with $\sigma$ satisfying $\sigma \geq 1$ and $\sigma \geq\left(1+\frac{\gamma_{-}}{p}\right)$.

Then, using the inequality of the type SNG in (2.3), with $r=\widetilde{\lambda}, s=\lambda_{0}$ and $\widetilde{p}=\beta+2$, we rewrite the inequality in (3.4) as

$$
\|w(\cdot, t)\|_{L^{\tilde{\lambda}}\left(\mathbb{R}^{n}\right)} \leq C\|w(\cdot, t)\|_{L^{\lambda}\left(\mathbb{R}^{n}\right)}^{(1-\theta)}\|\nabla w(\cdot, t)\|_{L^{(\beta+2)}\left(\mathbb{R}^{n}\right)}^{\theta},
$$

where $\theta$ and $(1-\theta)$ are giving by

$$
\begin{gathered}
\theta=\frac{n(q+\alpha+\beta)[(\sigma-1) q+\sigma \gamma]}{(q+\gamma)[q(\beta+2)+\sigma n(q+\alpha+\beta)-n q]} \\
(1-\theta)=\frac{[(q+\gamma)(\beta+2)-n \gamma+n(\alpha+\beta)]}{[q(\beta+2)+\sigma n(q+\alpha+\beta)-n q]} \frac{q}{(q+\gamma)} .
\end{gathered}
$$

After using (3.5) in (3.4) and substituting $\theta$ and $(1-\theta)$ by their respective values, we obtain

$$
\begin{gathered}
\frac{d}{d t}\|w(\cdot, t)\|_{L^{\lambda}\left(\mathbb{R}^{n}\right)}^{\lambda}+q(q-1)\left(\frac{(\beta+2)}{(q+\alpha+\beta)}\right){ }_{\lambda \frac{1}{n} \frac{[(q+\kappa)(\beta+1)+(n-1)(\alpha+\beta-\kappa)]}{\left[\left(\sigma-1+\frac{(\beta+2)}{n}\right) q+\sigma(\alpha+\beta)\right]}}^{(\beta+2)}\|\nabla w(\cdot, t)\|_{L^{\beta+2}\left(\mathbb{R}^{n}\right)}^{(\beta+2)} \leq \\
\leq q(q-1) B(t) \frac{(\beta+2)}{(q+\alpha+\beta)} C^{\frac{(\beta+1)(q+\gamma)}{(q+\alpha+\beta)}\|w(\cdot, t)\|_{L^{\lambda}\left(\mathbb{R}^{n}\right)}} \\
.\|\nabla w(\cdot, t)\|_{L^{(\beta+2)}\left(\mathbb{R}^{n}\right)}^{\left[\left(\sigma-1+\frac{(\beta+2)}{n}\right) q+\sigma(\alpha+\beta)\right]}
\end{gathered}
$$

Finally, rewriting (3.6) in terms of $u$, we arrive at

$$
\begin{aligned}
& \frac{d}{d t}\|u(\cdot, t)\|_{L^{q}\left(\mathbb{R}^{n}\right)}^{q}+q(q-1) \int_{\mathbb{R}^{n}}|u(x, t)|^{q-2+\alpha}|\nabla u(x, t)|^{\beta+2} d x \leq \\
& \leq q(q-1) B(t) \frac{(\beta+2)}{(q+\alpha+\beta)} \cdot C^{(\beta+1) \frac{(q+\gamma)}{(q+\alpha+\beta)}}\|u(\cdot, t)\|_{L^{q / \sigma}\left(\mathbb{R}^{n}\right)}^{\left.\left.q \cdot \frac{1}{n} \cdot \frac{[(q+\kappa)(\beta+1)+(n-1)(\alpha+\beta-\kappa)]}{n}\right) q+\sigma(\alpha+\beta)\right]} .
\end{aligned}
$$

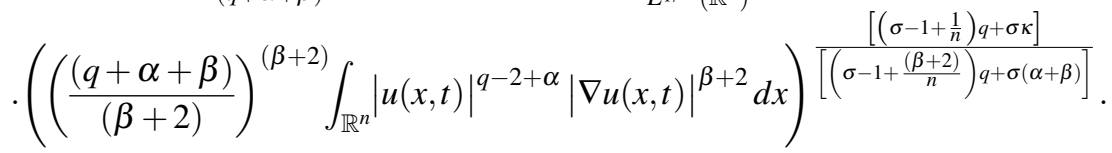


In the following we present the major result of this work: the determination of constraints on $\kappa \geq 0$ that guarantee the existence of global solutions to (3.1).

Theorem 3.4. Under the hypothesis of Theorems 2.1 and 2.3, the solutions to (3.1) satisfy:

(i) If $0 \leq \kappa<(\alpha+\beta)+\frac{(\beta+1)}{n}$, then $u(\cdot, t)$ is defined for all $0<t<\infty$ (given any initial $\left.u_{0}\right)$.

(ii) If $\kappa=(\alpha+\beta)+\frac{(\beta+1)}{n}$, the solutions are global when

$$
\left\|u_{0}\right\|_{L^{1}\left(\mathbb{R}^{n}\right)} \leq \mathbb{B}(0, \infty)^{\frac{-n}{(\beta+1)}} C^{-n \frac{2+(\alpha+\beta)+\frac{(\beta+2)}{(2+\alpha+\beta)}}{(2+\alpha+\beta}}\left(\frac{\beta+2}{2+,}\right.
$$

where $C$ is the constant in the SNG inequality used to obtain (3.2).

(iii) If $\kappa>(\alpha+\beta)+\frac{(\beta+1)}{n}$, the solutions are global when the initial $u_{0}$ satisfies

$$
\left\|u_{0}\right\|_{L^{1}\left(\mathbb{R}^{n}\right)}\left\|u_{0}\right\|_{L^{\infty}\left(\mathbb{R}^{n}\right)}^{\frac{n[\kappa-(\alpha+\beta)]-(\beta+1)}{(\beta+1)}} \leq\left\{\widetilde{C} \mathbb{B}(0 ; t)^{\frac{1}{(\beta+1)}}\right\}^{-n}
$$

where

$$
\widetilde{C}=\frac{\{2 n[\kappa-(\alpha+\beta)]+(\beta+1)(\alpha+\beta)\}}{(\beta+1)(\beta+2)} C^{\frac{\left[\left(2+\frac{1}{n}\right) n[\kappa-(\alpha+\beta)]+(\beta+1) \kappa\right]}{2 n[\kappa-(\alpha+\beta)]+(\beta+1)(\alpha+\beta)}}
$$

Proof. Case (i): $0 \leq \kappa<(\alpha+\beta)+\frac{(\beta+1)}{n}$.

By Theorem 2.3, taking $p=1$, we obtain

$$
\begin{aligned}
\|u(\cdot, t)\|_{L^{\infty}\left(\mathbb{R}^{n}\right)} \leq \widetilde{K} \max \{ & \left\|u\left(\cdot, t_{0}\right)\right\|_{L^{\infty}\left(\mathbb{R}^{n}\right)} \\
& \left.\left(\mathbb{B}\left(t_{0} ; t\right)\right)^{\frac{n}{n(\alpha+\beta-\kappa)+(\beta+1)}}\left(\mathbb{U}_{1}\left(t_{0} ; t\right)\right)^{\frac{(\beta+1)}{n(\alpha+\beta-\kappa)+(\beta+1)}}\right\} .
\end{aligned}
$$

By Theorem 2.1, we have that $\|u(\cdot, t)\|_{L^{1}\left(\mathbb{R}^{n}\right)} \leq\left\|u_{0}\right\|_{L^{1}\left(\mathbb{R}^{n}\right)}$. Then, it follows that

$$
\begin{aligned}
\|u(\cdot, t)\|_{L^{\infty}\left(\mathbb{R}^{n}\right)} \leq \widetilde{K} \max \{ & \left\|u\left(\cdot, t_{0}\right)\right\|_{L^{\infty}\left(\mathbb{R}^{n}\right)} ; \\
& \left.\left(\mathbb{B}\left(t_{0} ; t\right)\right)^{\frac{n}{n(\alpha+\beta-\kappa)+(\beta+1)}}\left\|u_{0}\right\|_{L^{1}\left(\mathbb{R}^{n}\right)}^{\frac{(\beta+1)+(\beta+1)}{n(\alpha+\beta-\kappa)}}\right\},
\end{aligned}
$$

for all $t \in\left(0, T_{*}\right)$. Therefore, the solution $u(\cdot, t)$ can be extended past $T_{*}$, and we conclude that solutions $u(\cdot, t)$ are limited for all $0<t<\infty$, i.e., the solution is global. This concludes case (i).

Case (ii): $\kappa=(\alpha+\beta)+\frac{(\beta+1)}{n}$.

In this case, we have

$$
\frac{n[\kappa-(\alpha+\beta)]}{(\beta+1)}=1
$$


and, therefore, the resctriction $p>\frac{n[\kappa-(\alpha+\beta)]}{(\beta+1)}$ does not allow the use of Theorem 2.3 with $p=1$, so that we cannot use the decrease of the norm $L^{1}$ of the solution, as in case (i).

For $\kappa$ in this case, we have

$$
\gamma=\frac{\left[(\alpha+\beta)+\frac{(\beta+1)}{n}\right](\beta+2)-(\alpha+\beta)}{(\beta+1)}=(\alpha+\beta)+\frac{(\beta+2)}{n} \geq 0
$$

and, therefore, $\gamma_{-}=0$, so that the conditions about $\sigma$ are satisfied for all $\sigma \geq 1$. We choose $\widehat{p}=1$, and, consequently, the restriction $q \geq 2$ makes it so that if $q \geq \sigma \widehat{p}$ is to be satisfied, then $\sigma \geq 2$. For simplicity, we use $\sigma=2$. With these choices, we use (3.2) to estimate $\|u(\cdot, t)\|_{L^{q}\left(\mathbb{R}^{n}\right)}=$ $\|u(\cdot, t)\|_{L^{2}\left(\mathbb{R}^{n}\right)}$. This is the norm we will be using in Theorem 2.3 to control $\|u(\cdot, t)\|_{L^{\infty}\left(\mathbb{R}^{n}\right)}$ in this case. Rewriting (3.2), using $p=1$ and $\sigma=2$, substituting $\kappa=(\alpha+\beta)+\frac{(\beta+1)}{n}$, and taking an upper limit to $B(t)$ on $(0, t)$, we arrive at

$$
\begin{aligned}
& \frac{d}{d t}\|u(\cdot, t)\|_{L^{2}\left(\mathbb{R}^{n}\right)}^{2}+2 \int_{\mathbb{R}^{n}}|u(x, t)|^{\alpha}|\nabla u(x, t)|^{\beta+2} d x \leq \\
& \leq\left(\frac{(2+\alpha+\beta)}{(\beta+2)}\right)^{(\beta+1)} C^{\frac{(\beta+1)(2+\gamma)}{(2+\alpha+\beta)}} \mathbb{B}(0, t)\left\|u_{0}\right\|_{L^{1}\left(\mathbb{R}^{n}\right)}^{\frac{(\beta+1)}{n}} \\
& .2 \int_{\mathbb{R}^{n}}|u(x, t)|^{\alpha}|\nabla u(x, t)|^{\beta+2} d x .
\end{aligned}
$$

Note that (3.10) informs that $\frac{d}{d t}\|u(\cdot, t)\|_{L^{2}\left(\mathbb{R}^{n}\right)}^{2} \leq 0$ for all $t \in(0, T) \backslash E_{q}$, provided that

$$
\left(\frac{(2+\alpha+\beta)}{(\beta+2)}\right)^{(\beta+1)} C^{(\beta+1) \frac{2+(\alpha+\beta)+\frac{(\beta+2)}{n}}{(2+\alpha+\beta)}} \mathbb{B}(0, t)\left\|u_{0}\right\|_{L^{1}\left(\mathbb{R}^{n}\right)}^{\frac{(\beta+1)}{n}} \leq 1,
$$

i.e., $\mathbb{B}(0, t)^{\frac{n}{(\beta+1)}}\left\|u_{0}\right\|_{L^{1}\left(\mathbb{R}^{n}\right)} \leq C^{-n \frac{2+(\alpha+\beta)+\frac{(\beta+2)}{n}}{(2+\alpha+\beta)}}\left(\frac{\beta+2}{2+\alpha+\beta}\right)^{n}$ for some $t>0$. In this cenario, then $\|u(\cdot, t)\|_{L^{2}\left(\mathbb{R}^{n}\right)}$ is decreasing in $(0, t)$, and therefore the solution to (3.1) is defined in $(0, t)$.

In particular, if (3.7) is satisfied, i.e.,

$$
\left\|u_{0}\right\|_{L^{1}\left(\mathbb{R}^{n}\right)} \leq \mathbb{B}(0, \infty)^{\frac{-n}{(\beta+1)}} C^{-n \frac{2+(\alpha+\beta)+\frac{(\beta+2)}{(2+\alpha+\beta)}}{n}}\left(\frac{\beta+2}{2+\alpha+\beta}\right)^{n},
$$

then the solution to (3.1) is globally defined (i.e., $T_{*}=\infty$ ). This concludes case (ii).

Case (iii): $\kappa>(\alpha+\beta)+\frac{(\beta+1)}{n}$.

In this case, we choose

$$
\widehat{p}=\frac{n[\kappa-(\alpha+\beta)]}{(\beta+1)} .
$$

Again, it is not possible to apply Theorem 2.3 directly because $\widehat{p}$ does not satisfy the condition $p>\frac{n(\kappa-(\alpha+\beta))}{(\beta+1)}$. Besides, with the values of $\kappa$ for this case, we have that $\widehat{p}>1$, meaning we cannot use the decrease of norm $L^{1}$ of the solution. As in case (ii), the values for $\kappa$ in this case 
lead us to $\gamma_{-} \geq 0$, therefore, the conditions on $\sigma$ are satisfied by any $\sigma \geq 1$. For simplicity, we use again $\sigma=2$.

With these choices for $\widehat{p}$ and $\sigma$, we try to use Theorem 2.3 to control the norm of the sup of the solution $u(\cdot, t)$ by its $L^{q}$ norm, with $q=\sigma \widehat{p}=2 \widehat{p}$.

Rewriting the inequality (3.2) using the chosen values for $\widehat{p}$ and $\sigma$, substituting $\gamma$ and taking the sup on $(0, t)$ of $B(t)$ as an upper limit to it, we obtain

$$
\begin{gathered}
\frac{d}{d t}\|u(\cdot, t)\|_{L^{2 \widehat{p}}\left(\mathbb{R}^{n}\right)}^{2 \widehat{p}}+2 \widehat{p}(2 \widehat{p}-1) \int_{\mathbb{R}^{n}}|u(x, t)|^{2 \widehat{p}-2+\alpha}|\nabla u(x, t)|^{\beta+2} d x \leq \\
\leq\left(\frac{2 \widehat{p}+\alpha+\beta}{\beta+2}\right)^{(\beta+1)} C^{(\beta+1) \frac{\left[\left(2+\frac{1}{n}\right) n[\kappa-(\alpha+\beta)]+(\beta+1) \kappa\right]}{2 n[\kappa-(\alpha+\beta)]+(\beta+1)(\alpha+\beta)}} \mathbb{B}(0 ; t)\|u(\cdot, t)\|_{L^{\widehat{p}}\left(\mathbb{R}^{n}\right)}^{\widehat{p}(\beta+1)} \\
.2 \widehat{p}(2 \widehat{p}-1) \int_{\mathbb{R}^{n}}|u(x, t)|^{2 \widehat{p}-2+\alpha}|\nabla u(x, t)|^{\beta+2} d x .
\end{gathered}
$$

As in case (ii), (3.12) would give us $\frac{d}{d t}\|u(\cdot, t)\|_{L^{2 \widehat{p}\left(\mathbb{R}^{n}\right)}}^{2 \widehat{x}} \leq 0, \forall t \in(0, T) \backslash E_{q}$, provided that

$$
\left(\frac{2 \widehat{p}+\alpha+\beta}{\beta+2}\right)^{(\beta+1)} C^{(\beta+1) \frac{\left[\left(2+\frac{1}{n}\right) n[\kappa-(\alpha+\beta)]+(\beta+1) \kappa\right]}{2 n[\kappa-(\alpha+\beta)]+(\beta+1)(\alpha+\beta)}} \mathbb{B}(0 ; t)\|u(\cdot, t)\|_{L^{\widehat{p}}\left(\mathbb{R}^{n}\right)}^{\widehat{(\beta+1)}} \leq 1,
$$

i.e.,

$$
\widetilde{C} \mathbb{B}(0 ; t)^{\frac{1}{(\beta+1)}}\|u(\cdot, t)\|_{L^{\widehat{p}}\left(\mathbb{R}^{n}\right)}^{\frac{\hat{p}}{n}} \leq 1,
$$

where $\widetilde{C}=\frac{\{2 n[\kappa-(\alpha+\beta)]+(\beta+1)(\alpha+\beta)\}}{(\beta+1)(\beta+2)} C^{\frac{\left[\left(2+\frac{1}{n}\right) n[\kappa-(\alpha+\beta)]+(\beta+1) \kappa\right]}{2 n[\kappa-(\alpha+\beta)]+(\beta+1)(\alpha+\beta)}}$.

The condition (3.13) is sufficient to guarantee that $\|u(\cdot, t)\|_{L^{2} \widehat{p}\left(\mathbb{R}^{n}\right)}$ is decreasing (and therefore controls, by Theorem2.3, the sup norm). The problem is, up to this moment, we do not control $\|u(\cdot, t)\|_{L^{\widehat{p}\left(\mathbb{R}^{n}\right)}}$.

To find out what is necessary so that (3.13) is satisfied, we proceed as follows: we use the interpolation of norms inequatily to obtain

$$
\|u(\cdot, t)\|_{L^{\widehat{p}}\left(\mathbb{R}^{n}\right)} \leq\|u(\cdot, t)\|_{L^{1}\left(\mathbb{R}^{n}\right)}^{\frac{1}{2 \widehat{P}-1}}\|u(\cdot, t)\|_{L^{2} \vec{p}\left(\mathbb{R}^{n}\right)}^{\frac{2(\widehat{p}-1)}{2 \hat{-1}}},
$$

we raise both sides of(3.14) to $\frac{\widehat{p}}{n}$ power, and multiply by $\widetilde{C} \mathbb{B}(0 ; t)^{\frac{1}{(\beta+1)}}$, with $\widetilde{C}$ as in(3.9), to obtain

$$
\widetilde{C} \mathbb{B}(0 ; t)^{\frac{1}{(\beta+1)}}\|u(\cdot, t)\|_{L^{\frac{\hat{p}}{n}}\left(\mathbb{R}^{n}\right)}^{\frac{\hat{p}}{C}} \leq \widetilde{C} \mathbb{B}(0 ; t)^{\frac{1}{(\beta+1)}}\|u\|_{L^{1}\left(\mathbb{R}^{n}\right)}^{\frac{\hat{p}}{n(2 \widehat{n}-1)}}\|u\|_{L^{2 \widehat{p}}\left(\mathbb{R}^{n}\right)}^{\frac{2 \widehat{p}(\widehat{p}-1)}{n(2-1)}} .
$$

Then we use again the interpolation of norms inequality to estimate $\|u(\cdot, t)\|_{L^{2 \widehat{p}}}$, i.e.,

$$
\|u(\cdot, t)\|_{L^{2} \widehat{p}\left(\mathbb{R}^{n}\right)} \leq\|u(\cdot, t)\|_{L^{1}\left(\mathbb{R}^{n}\right)}^{\frac{1}{2 \widehat{C}}}\|u(\cdot, t)\|_{L^{\infty}\left(\mathbb{R}^{n}\right)}^{\frac{2 \widehat{p}-1}{2 \hat{r}}},
$$

and, substituting (3.16) in (3.15), we obtain

$$
\widetilde{C} \mathbb{B}(0 ; t)^{\frac{1}{(\beta+1)}}\|u(\cdot, t)\|_{L^{\hat{p}}\left(\mathbb{R}^{n}\right)}^{\frac{\widehat{p}}{n}} \leq \widetilde{C} \mathbb{B}(0 ; t)^{\frac{1}{(\beta+1)}}\|u(\cdot, t)\|_{L^{1}\left(\mathbb{R}^{n}\right)}^{\frac{1}{n}}\|u(\cdot, t)\|_{L^{\infty}\left(\mathbb{R}^{n}\right)}^{\frac{(\widehat{p}-1)}{n}} .
$$


We claim that, if we make $u_{0} \in L^{1}\left(\mathbb{R}^{n}\right) \cap L^{\infty}\left(\mathbb{R}^{n}\right)$ satisfy the condition (3.8), rewritten as

$$
\widetilde{C} \mathbb{B}(0 ; t)^{\frac{1}{(\beta+1)}}\left\|u_{0}\right\|_{L^{1}\left(\mathbb{R}^{n}\right)}^{\frac{1}{n}}\left\|u_{0}\right\|_{L^{\infty}\left(\mathbb{R}^{n}\right)}^{\frac{(\widehat{p}-1)}{n}} \leq 1
$$

where $\widetilde{C}$ is given in (3.9) and $\widehat{p}$ in (3.11), we will obtain

$$
\widetilde{C} \mathbb{B}(0 ; t)^{\frac{1}{(\beta+1)}}\|u(\cdot, t)\|_{L^{1}\left(\mathbb{R}^{n}\right)}^{\frac{1}{n}}\|u(\cdot, t)\|_{L^{\infty}\left(\mathbb{R}^{n}\right)}^{\frac{(\widehat{p}-1)}{n}} \leq 1,
$$

at least for $t \in\left(0, T_{*}\right)$ close to zero. But this is sufficient to guarantee as well that (3.18) is valid for any $t \in\left(0, T_{*}\right)$. Indeed, if (3.18) was not true for any $t \in\left(0, T_{*}\right)$, then there would exists $T_{1}$, with $0<T_{1}<T_{*}$, for which

$$
\begin{gathered}
\widetilde{C} \cdot \mathbb{B}(0 ; t)^{\frac{1}{(\beta+1)}}\|u(\cdot, t)\|_{L^{1}\left(\mathbb{R}^{n}\right)}^{\frac{1}{n}}\|u(\cdot, t)\|_{L^{\infty}\left(\mathbb{R}^{n}\right)}^{\frac{(\widehat{p}-1)}{n}} \leq 1, \quad \forall t \in\left(0, T_{1}\right), \\
\widetilde{C} \cdot \mathbb{B}(0 ; t)^{\frac{1}{(\beta+1)}}\left\|u\left(\cdot, T_{1}\right)\right\|_{L^{1}\left(\mathbb{R}^{n}\right)}^{\frac{1}{n}}\left\|u\left(\cdot, T_{1}\right)\right\|_{L^{\infty}\left(\mathbb{R}^{n}\right)}^{\frac{(\widehat{p}-1)}{n}}>1 .
\end{gathered}
$$

However, if (3.19) is valid, by (3.17) it follows that the inequality in (3.13) is valid for any $t \in\left(0, T_{1}\right)$ and, therefore, (3.12) implies that $\|u(\cdot, t)\|_{L^{2 \widehat{p}\left(\mathbb{R}^{n}\right)}}$ is decreasing, for all $t \in\left(0, T_{1}\right) \backslash E_{q}$. Then, by Theorem 2.3, $\|u(\cdot, t)\|_{L^{2 \hat{p}}\left(\mathbb{R}^{n}\right)}$ controls the norm of the sup, for all $t \in\left(0, T_{1}\right)$. As we have let $u_{0}$ satisfy (3.8), and both the norm $L^{1}$ and the norm $L^{\infty}$ decrease in $\left(0, T_{1}\right)$ (the norm $L^{\infty}$ decreases in $\left(0, T_{1}\right)$ by (3.19) and by the decrease of the norm $\left.L^{1}\right)$, by the continuity of the solution, if is not possible that we have (3.20).

Therefore, the inequality (3.18) is valid for all $t \in\left(0, T_{*}\right)$, and then (3.13) is also satisfied for all $t \in\left(0, T_{*}\right)$. We conclude by (3.12) that $\|u(\cdot, t)\|_{L^{2} \widehat{p}\left(\mathbb{R}^{n}\right)}$ decreases monotonically.

We then conclude, by Theorem 2.3, that the solution exists for all $t \in(0, \infty)$, provided that (3.8) is satisfied.

This ends the proof of Theorem 3.4.

\section{CONCLUSION}

In this work we consider the initial-value problem (1.1) for an doubly nonlinear advectiondiffusion equation and, with $p_{0}=1$, we presented conditions over $\kappa$ in (1.2) with which the existence of global solutions is guaranteed. In the general case where the sup norm is controlled in a specific form (see [2]), as an application of Theorems 2.1 and 2.3, in our main result, Theorem 3.2 (Section 3), we guaranteed that the condition over $\kappa$ for which the global existence of solution is always obtained is $0 \leq \kappa<(\alpha+\beta)+\frac{(\beta+1)}{n}$, i.e, independent of the initial profile $u_{0}$, for these valors of $\kappa$ the solutions are globals $\left(T_{*}=\infty\right)$. Even if $\kappa=(\alpha+\beta)+\frac{(\beta+1)}{n}$ or $\kappa \geq(\alpha+\beta)+\frac{(\beta+1)}{n}$, we presented conditions over $u_{0}$ for which the global solutions yet are possible. 


\title{
ACKNOWLEDGEMENTS
}

This work was partly supported by CNPq, Brazil.

\begin{abstract}
RESUMO. Neste trabalho, consideramos um problema de valor inicial para uma equação de advecção-difusão duplamente não linear, e apresentamos um valor crítico de $\kappa$ até o qual o problema de valor inicial tem solução global independente do dado inicial $u_{0}$, e a partir do qual as soluções globais ainda podem existir, mas para dados iniciais $u_{0}$ satisfazendo a determinadas condições. Para isso, supomos que a função $f(x, t, u)$ no termo advectivo, escrito na forma divergente, satisfaz a certas condições a respeito de sua variação em $\mathbb{R}^{n}$, e usamos também o decrescimento na norma $L^{1}\left(\mathbb{R}^{n}\right)$ e um controle para a norma $L^{\infty}\left(\mathbb{R}^{n}\right)$ da solução $u(\cdot, t)$.
\end{abstract}

Palavras-chave: equações parabólicas duplamente não lineares, soluções globais, condições para soluções globais.

\section{REFERENCES}

[1] J.Q. Chagas. "Contribuições para a teoria de equações parabólicas duplamente não lineares com termos advectivos”. Ph.D. thesis, UFRGS, Universidade Federal do Rio Grande do Sul, Porto Alegre, RS (2015).

[2] J.Q. Chagas, P.L. Guidolin \& P.R. Zingano. Norma do sup para equações de advecção-difusão duplamente não lineares: um caso de decrescimento. In "Proceeding Series of the Brazilian Society of Applied and Computational Mathematics", 5, v.1. SBMAC (2017). doi:10.5540/03.2017.005.01.0034.

[3] J.Q. Chagas, P.L. Guidolin \& P.R. Zingano. Norma do sup para equações de advecção-difusão duplamente não lineares: o caso geral. In "Proceeding Series of the Brazilian Society of Applied and Computational Mathematics", 6, v.2. SBMAC (2018). doi:10.5540/03.2018.006.02.0273.

[4] E. DiBenedetto. "Degenerate Parabolic Equations". Springer-Verlag, New York (1993).

[5] P.B. e Silva, L. Schütz \& P. Zingano. On some energy inequalities and supnorm estimates for advectiondiffusion equations in $R^{n}$. Nonlinear Analysis: Theory, Methods \& Applications, 93 (2013), 90-96. doi:10.1016/j.na.2013.07.028.

[6] A. Friedman. "Partial Differential Equations". Holt, Rinehart and Winston, New York (1969).

[7] O. Ladyzhenskaya, V.A. Solonnikov \& N.N. Uralceva. "Linear and Quasilinear Equations of Parabolic Type". American Mathematical Society, Providence (1968).

[8] D. Serre. "Systems of Conservation Laws", volume 1. Cambridge University Press, Cambridge (1999).

[9] Z. Wu, J. Zhao, J. Yin \& H. Li. "Nonlinear Diffusion Equations”. World Scientific, Singapore (2001). 